\title{
Functional outcome of joint mobilization added to task-oriented training on hand function in chronic stroke patients
}

\author{
Asmaa Sabbah', Sherine El Mously ${ }^{2 *}$, Hanan Helmy Mohamed Elgendy ${ }^{1}$, Mona Adel Abd Eltawab Farag ${ }^{3}$ and \\ Abeer Abo Bakr Elwishy ${ }^{3}$
}

\begin{abstract}
Background: Approximately half of stroke patients show impaired upper limb and hand function. Task-oriented training focuses on functional tasks, while joint mobilization technique aims to restore the accessory movements of the joints.

Objective: To investigate the effect of adding joint mobilization to task-oriented training to help the patients in reaching a satisfactory level of recovery for their hand function.

Patients and methods: Thirty chronic stroke patients with paretic hand participated in the study; they were divided equally into study and control groups. The study group received joint mobilization followed by taskoriented training for the affected hand. Meanwhile, the control group received task-oriented training only. Both groups received their treatment in the form of 3 sessions per week for 6 successive weeks. The primary outcome measures were hand function that was assessed by Jebsen-Taylor hand function test (JTT) and active and passive wrist extension range of motion (ROM) that was measured by a standard goniometer. The secondary outcome measure was the grip strength of the hand that was assessed by a JAMAR adjustable hand dynamometer.
\end{abstract}

Results: There was a significant improvement in all the outcome measurements in both groups that were more evident in the study group.

Conclusion: Combining joint mobilization with task-oriented training had a highly significant effect in improving the hand function in chronic stroke patients compared to task-oriented training alone.

Keywords: Stroke, Spasticity, Hand function, Joint mobilization, Task-oriented training

\section{Introduction}

Stroke is defined as a neurological deficit attributed to an acute vascular focal injury of the central nervous system [1]. It is a worldwide common disease that leads to serious disabilities [2]. Hemiparesis is the most common motor impairment after a stroke and frequently leads to persistent hand dysfunction [3]. Nearly about $50 \%$ of stroke patients show impaired upper limb and hand

\footnotetext{
* Correspondence: smm02@fayoum.edu.eg

${ }^{2}$ Faculty of Medicine, Fayoum University, Keman Fares area, Fayoum City 63611, Egypt

Full list of author information is available at the end of the article
}

function and up to $74 \%$ rely on long-term help to perform their activities of daily living (ADL) $[4,5]$. The hand functions are complex as we use our hands in a vast variety of tasks such as grasping, pushing, holding objects, and expressing emotions [6].

Task-oriented training is a type of physiotherapy that encourages the active participation and focuses on functional tasks rather than simple repetitive training of normal motion patterns [7]. Joint mobilizations are used as an intervention to improve the range of motion (ROM), decreasing pain, and ultimately improving the upper extremity functions [8]. Joint mobilization technique 
proposed by Maitland is based on a graded system and is intended to restore the accessory movements of the joints by performing passive, rhythmic, and oscillatory movements [9].

After stroke, reduced ROM at joints occurs and it can be complicated by joint contractures. This occurs due to many factors such as reduced muscle length and increased stiffness of muscle and/or connective tissue. Such post stroke consequences can be solved by moving the joints through a full ROM with pressure at the end of range using the manual therapy [10]. Mobilization may help stroke patients in reducing the joint stiffness [11]. Moreover, it provides afferent input that can be used in facilitating the motor activity $[12,13]$. Accordingly, we aimed to investigate the effect of adding joint mobilization to task-oriented training in order to help those patients in reaching a satisfactory level of recovery for their hand functions.

\section{Patients and methods}

This cross-section case control study was conducted in the period from March 2017 to December 2018. Thirty patients diagnosed clinically and radiologically with ischemic stroke were recruited from the stroke outpatient clinic of El Kasr El Ainy hospitals. The inclusion of ischemic stroke only was made aiming to unify the underlying pathology in an attempt to standardize the selected study population and to exclude any possible different etiological sequelae. All included patients developed a stroke at least 6 months before the time of the study. They were right-handed, and their age ranged from 45 to 60 years old. They had spasticity grade 1 and +1 according to Modified Ashworth Scale (MAS) [14]. Only patients who got $\geq 24$ points on the mini mental state examination (MMSE) [15]were included, suggesting sufficient cognitive and communication abilities to understand the study and to give consent. Left-handed patients, those with history of stroke onset less than 6 months ago and those having dysfunction due to musculoskeletal disorders, were excluded. Also, patients receiving treatment for spasticity such as botulinum toxin or baclofen pump for up to 6 months and who were concurrently participating in other hand training program were excluded.

Patients were assigned randomly using the chit pick box method into two equal groups (study and control). The study group was treated with joint mobilization followed by task-oriented training for the affected hand. Meanwhile, the control group was treated with taskoriented training only. The treatment program was applied in the physiotherapy outpatient clinic of El Kasr El Ainy hospitals.

Concerning the patients' assessment, the JebsenTaylor hand function test (JTT) was used to evaluate the gross and fine motor functional hand abilities that reflect their ADL. It consists of simple and inexpensive materials. These subtests consist of writing a standardized sentence, turning over cards, picking up small objects (coins, paper clips, bottle caps), and placing them in an empty coffee can, simulated feeding, stacking checkers, moving large light objects (empty baked beans cans), and moving large heavy objects (full baked beans cans) [16]. The JTT takes 10 to $15 \mathrm{~min}$ to be completed. The time to complete each subtest is measured in seconds, with longer time to completion indicating greater impairment in hand functions [17].

Patients were seated directly on a chair in front of a table and were provided with instructions and a demonstration of the subtests. All subtests were administered in precisely the same manner to all patients who were instructed to perform the tasks as rapidly and accurately as possible according to written standardized instructions in the testing set [16]. The total JTT time and partial subtest JTT times were measured using a stopwatch. The writing task was not included. For each subset, 3 trials were recorded for each participant, and the average was used for analysis.

To measure the ROM of active and passive wrist extension, we used the standard goniometer. It has two arms; one is fixed while the other one is moving. It has one dial for reading degree of motion and one axis around which other arms revolve. Axis is placed on joint line. The limb of which the ROM is to be measured was placed along the moving arm. Once the required range was achieved, reading was obtained [18]. The reliability of this test is shown with an intraclass correlation coefficient of 0.95 [19].

The patient sits on a chair with the forearm positioned on the table or on the arm support of the chair with the elbow in 90-degree flexion and in full pronation with the hand over the edge of the table or arm support. Then, the ROM for active and passive wrist extension was measured. The bony prominences (triquetrum, olecranon, and fifth metacarpal head) were marked to identify the reference points clearly. The center of the goniometer was placed at the center of the axis of the wrist joint (triquetrum bone), with one arm of the goniometer placed parallel to the lateral midline of the ulna and a moveable arm of the goniometer placed to the lateral midline of the fifth metacarpal bone. Values were recorded 3 times for each patient, and the average was used for analysis.

Then, the JAMAR adjustable hand dynamometer (USA, serial number 30402340) was applied to measure the grip strength of the paretic hand. It exhibits excellent test-retest reliability $(0.97-0.99)$ [20]. It has dual scale readout as it displays a grip force up to $200 \mathrm{lbs}$ or $90 \mathrm{~kg}$ of maximum reading. For convenience and easy 
recording, the JAMAR automatically retains the highest reading on special peak hold needle. It is isometric in use with almost no predictable motion of handles regardless of the grip strength. The hand grasp is comfortable and effective. These features are combined to ensure accurate reproducible results. It has an adjustable handle to accommodate various hands' size and it can adjust to give five grip positions. The strength of the hand grip is affected by different handle positions; strength is the greatest for the middle handle position and drop for the wider and narrower positions.

The patient was asked to hold the upper arm vertically to the side of the trunk, forearm horizontal with elbow $90^{\circ}$ flexion, and wrist was positioned between $0^{\circ}$ and $30^{\circ}$ extension and between $0^{\circ}$ and $15^{\circ}$ ulnar deviation. The JAMAR adjustable hand dynamometer was set to the middle handle position. The peak hold needle was rotated counter clockwise to $0^{\circ}$. The patient arranged the instrument in his hand; then, he was asked to squeeze as hard as possible. After $3 \mathrm{~s}$ of continuous compression, the patient was asked to relax. The highest force exerted was automatically recorded by the peak hold needle. The reading was recorded, and the peak hold needle was reset to zero. The scores of 3 successive trials were recorded, and the mean was calculated and reported.

Regarding the treatment program, the study group received $20 \mathrm{~min}$ of joint mobilization followed by $40 \mathrm{~min}$ of task-oriented training for the affected hand. Meanwhile, the control group received $40 \mathrm{~min}$ of taskoriented training only for the affected hand. Both groups received 3 sessions per week (every other day) for 6 successive weeks.

The task-oriented training rehabilitation program was done following the guidelines of Carr and Shepherd [21]. In the present study, the patient rather than the therapist selected the tasks to be included in the training. The tasks were important to the patients in their ADL and would have a good impact on the patient's sense of satisfaction. Six tasks that match the patients' training preferences were chosen from 2 exercise lists (3 from each list). The first list consists of five tasks: grasping, transferring and releasing a can, stacking plastic cups, screwing and unscrewing a lid, pressing door handle to open a door, and pouring water from one container to another, while the second list consists of another five tasks: using a key to unlock or lock a door, turning over the page of book, using a spoon, stirring coffee, and finally, buttoning and unbuttoning.

Joint mobilization grade 3 (through the range) and grade 4 (at the end of the range) were applied to gain the ROM and to restore the optimal kinematics between the joint surfaces [22]. This program consists of inferior radioulnar joint supination, wrist extension, wrist radial deviation, radiocarpal extension, radiocarpal supination, metacarpophalangeal joint extension, and first carpometacarpal joint extension. The oscillatory movements performed during the mobilization were applied at a rate of 2-3 oscillations per second and lasted approximately 2 min for each movement direction [23, 24].

\section{Statistical analysis}

Descriptive statistics and $t$ test were conducted for comparison of the patients' characteristics between both groups. Chi-squared test was conducted for comparison of affected hand, sex, and MAS spasticity grades' distribution between both groups. Mixed multivariate analysis of variance (MANOVA) was conducted to compare the effect of time (pre versus post) and the effect of treatment between both groups, as well as the interaction between time and treatment on mean values of JTT, active and passive wrist extension ROM, and grip strength. The level of significance was set at $p<0.05$, and $p<$ 0.001 was considered highly significant. Statistical analysis was performed through the Statistical Package for Social Science (SPSS) version 22 for windows.

\section{Results}

The study included thirty patients who were divided equally into study and control groups. The study group included $6(40 \%)$ females and $9(60 \%)$ males while the control group included 7 (47\%) females and 8 (53\%) males with no statistically significant difference between both groups in the sex distribution $(p=0.71)$. Both groups included $6(20 \%)$ patients presented with faciobrachial monoplegia, 4 on the left side and 2 on the right side. Meanwhile, $24(80 \%)$ are presented with hemiparesis, 14 on the left side and 10 on the right side. Concerning the affected hand distribution, the study group included $4(27 \%)$ patients with right hand affection and $11(73 \%)$ with left hand affection. Meanwhile, the control group included $6(40 \%)$ patients with right hand affection and $9(60 \%)$ with left hand affection. There was no statistically significant difference between both groups in the distribution of the affected hand $(p=0.43)$.

General characteristics of the patients of both groups are exhibited in Table 1 . There was no statistically

Table 1 General characteristics of patients of the study and control groups

\begin{tabular}{llll}
\hline & $\begin{array}{l}\text { Study group } \\
\text { Mean } \pm \text { SD }\end{array}$ & $\begin{array}{l}\text { Control group } \\
\text { Mean } \pm \text { SD }\end{array}$ & $\begin{array}{l}\boldsymbol{p} \\
\text { value }\end{array}$ \\
\hline Age (years) & $55.53 \pm 4.73$ & $54.66 \pm 5.19$ & 0.63 \\
Weight $(\mathbf{k g})$ & $86.13 \pm 5.64$ & $84.86 \pm 9.84$ & 0.66 \\
Height (cm) & $173.2 \pm 8.1$ & $171.4 \pm 8.65$ & 0.56 \\
Duration of illness (month) & $27 \pm 6.52$ & $28.06 \pm 7.5$ & 0.68 \\
MMSE & $27.86 \pm 1.35$ & $28.2 \pm 1.42$ & 0.51 \\
\hline
\end{tabular}

SD standard deviation, $M D$ mean difference, $\mathrm{kg}$ kilogram, $\mathrm{cm}$ centimeter 
significant difference in the general characteristics and in the MAS spasticity grades' distribution between the groups $(p>0.05)$ as shown in Tables 1 and 2.

Regarding the effect of treatment on JTT, there was a significant decrease in the time needed to complete its subsets (turning over cards, picking up small objects, simulated feeding, stacking checkers, moving large light objects, and moving large heavy objects) in the study group posttreatment compared with pre-treatment ( $p=0001$ for all the subsets). Similar results were obtained in the control group post-treatment compared with pre-treatment $(p=$ $0.0001,0.02,0.0001,0.01,0.01$, and 0.03 respectively).

The multiple pairwise comparison showed that there was no significant difference in the time needed to complete the JTT subsets between the study and control groups ( $p>0.05$ for all the subsets). However, there was a significant decrease in the mean values of these subsets in the study group post-treatment compared with that of control group $(p=0.01,0.003,0.001,0.0001,009$, and 0.03 respectively).

When we studied the total JTT time, there was a significant decrease in total JTT time in either the study group and the control group post-treatment compared with pre-treatment $(p=0.0001)$. Multiple pairwise comparison showed that there was no significant difference in the total JTT time pre-treatment between the study and control groups $(p=0.19)$. However, there was a significant decrease in the mean values of the total JTT time of the study group post-treatment compared with that of control group $(p=0.0001)$.

Regarding the effect of treatment program on active, passive wrist extension ROM, and hand grip strength, the study group showed a significant increase in the posttreatment compared with the pre-treatment $(p=0.0001)$. Furthermore, the control group exhibited similar results post-treatment compared with the pre-treatment ( $p=$ $0.004,0.004$, and 0.001 respectively). The multiple pairwise comparison between both groups showed no significant difference in these tests pre-treatment $(p>0.05$ respectively). However, a significant increase in the mean values of these tests was noticed post-treatment compared with those of control group ( $p=0.0001,0.0001$, and 0.001 respectively). These results are illustrated in Fig. 1.

\section{Discussion}

The current study aimed to investigate the effect of adding joint mobilization to task-oriented training on hand

Table 2 Comparison of MAS spasticity grades' distribution between study and control groups

\begin{tabular}{llcll}
\hline & Study group & Control group & $\boldsymbol{x}^{\mathbf{2}}$ & $\boldsymbol{p}$ value \\
\hline Grade $\mathbf{1}$ & $8(53 \%)$ & $10(67 \%)$ & 0.55 & 0.45 \\
Grade $+\mathbf{1}$ & $7(47 \%)$ & $5(33 \%)$ & & \\
\hline
\end{tabular}

$x^{2}$ chi-squared value function in chronic stroke patients. The hand function was assessed by the JTT, the active and passive wrist extension ROM was measured by the standard goniometer, and the grip strength of the paretic hand was assessed by a JAMAR adjustable hand dynamometer. All the measurement outcomes were assessed before starting and after applying a 6-week treatment program for all patients. The primary outcome measures were the hand function added to the active and passive wrist extension ROM. The secondary outcome measure was the grip strength.

The results showed a significant improvement in the JTT (total time and partial subtest times), active and passive wrist extension ROM, and hand grip strength in both groups post-treatment. However, there was a significant difference between both groups regarding these improvements in favor to the study group.

Our results come in agreement with Park and Youn [13] who conducted a study to confirm the effect of wrist joint mobilization with taping on the ROM, grip strength, and spasticity in stroke patients. The results of their study showed that wrist joint mobilization with taping has a significant effect in ROM, grip strength, and spasticity, whereas there was no significant difference in the control group with just taping.

Smedes and colleagues [12] conducted a study to investigate the effects of manual mobilization of the wrist in the rehabilitation of chronic hemiplegic hand after stroke. The primary outcome measures were wrist extension ROM and activity limitation. The secondary outcome measures were spasticity, grip strength, and pain. The results showed that mobilization of the wrist has a significant beneficial influence on the recovery of the wrist's mobility, activity limitation, and the grip strength. The results of our study can be explained by the positive relationship that was demonstrated between the improvement in ROM and the improvement in hand function in stroke patients [12].

It was reported that post-stroke patients experience stiffness which represents a major challenge to perform tasks and makes attempts at using the affected arm extremely difficult [3]. Joint mobilization techniques have specially increasing ROM [18]. Maitland mobilization improves ROM by producing mechanical effects that involve a permanent or temporary change in the length of connective tissue structures such as joint capsule, ligaments, and muscle [25]. Applying joint mobilization techniques to limited motion joint in the stroke patients were found to be effective in improving the ROM. Thus, the increase in available motion could result in improving the efficiency of movement and decrease the time needed to perform the tasks [26-28].

It is also possible that the improvement in joint flexibility would allow the muscles to increase their action. 


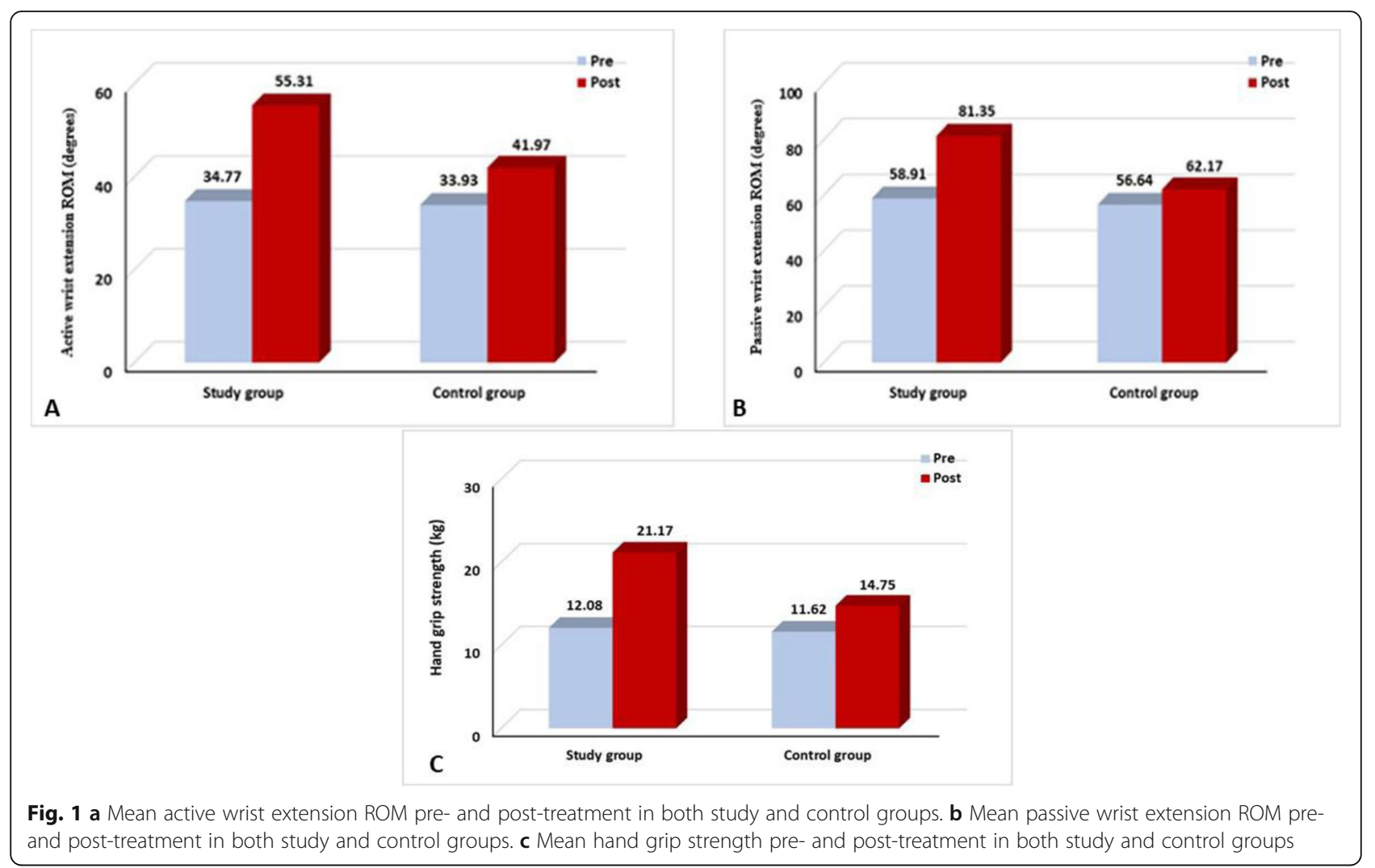

In addition, mobilization appears to be an appropriate and effective interventional therapy that potentially will kick start the process of activating muscle after stroke by providing significant proprioceptive information to the brain, facilitating direct activation of the primary motor cortex and the corticospinal system to increase the motor activity [29].

Limitations of this study were the small sample size. Also, the long-term follow-up was not considered to evaluate the duration of improvement; thus, in case of deterioration in the patients' performance, this would be missed. Further researches to investigate the long-term effects of mobilization in stroke patients are suggested. Moreover, it will be valuable to apply neurophysiologic assessments such as the functional magnetic resonance imaging for better understanding of the effect of mobilization in stroke rehabilitation.

\section{Conclusion}

Combining joint mobilization and task-oriented training had a highly significant effect in improving the paretic hand function in chronic stroke patients compared to the task-oriented training alone.

\section{Abbreviations}

ADL: Activities of daily living; $\mathrm{cm}$ : Centimeter; JTT: Jebsen-Taylor hand function test; kg: Kilogram; MANOVA: Multivariate analysis of variance; MAS: Modified Ashworth Scale; MD: Mean difference; MMSE: Mini mental state examination; ROM: Range of motion; SD: Standard deviation; SPSS: Statistical Package for Social Science

\section{Acknowledgements}

Not applicable.

\section{Authors' contributions}

ABE was responsible for planning the study design and supervising the correct implementation of the physiotherapy techniques applied on the selected study population. HHE was responsible for planning the study design and supervising the correct and precise selection of the stroke patients, reviewing the patient investigations, confirming the diagnosis, and performing the statistical analysis of the collected data. AS was responsible for performing the neurological assessment of the selected stroke patients and contributed to the scientific writing of the manuscript. MAF was responsible for applying the study protocol and assessment of the selected included patients and following them up. SM helped in writing the manuscript and she is responsible for the publication. The author(s) read and approved the final manuscript.

\section{Funding}

No funding was obtained from any institution for our study.

\section{Availability of data and materials}

Data can be available for publication only by special approval from Cairo University.

\section{Ethics approval and consent to participate}

The study was approved by the Ethical Committee of the Faculty of Physical Therapy, Cairo University (No. P.T.REC/012/001491). All patients were informed about the aim of the study, physical therapy program, its duration, and signed the informed consent form before starting the study. The confidentiality of their information was respected and their right not to participate in the study was ensured. 


\section{Consent for publication}

Not applicable.

\section{Competing interests}

The authors declare that they have no competing interests.

\section{Author details}

${ }^{1}$ Faculty of Medicine, Cairo University, Giza, Egypt. ${ }^{2}$ Faculty of Medicine, Fayoum University, Keman Fares area, Fayoum City 63611, Egypt. ${ }^{3}$ Department of Neuromuscular Disorders and Its Surgery, Faculty of Physical Therapy, Cairo University, Giza, Egypt.

Received: 18 January 2020 Accepted: 17 March 2020

Published online: 05 April 2020

\section{References}

1. Sacco RL, Kasner SE, Broderick JP, Caplan LR, Connors J, Culebras A, et al. An updated definition of stroke for the 21st century: a statement for healthcare professionals from the American Heart Association/American Stroke Association. Stroke. 2013;44:2064-89.

2. Le Q, Qu Y, Tao Y, Zhu S. Effects of repetitive transcranial magnetic stimulation on hand function recovery and excitability of the motor cortex after stroke: a meta-analysis. Am J Phys Med Rehabil. 2014:93(5):422-30.

3. Sabini RC, Dijkers MP, Raghavan P. Stroke survivors talk while doing: development of a therapeutic framework for continued rehabilitation of hand function post stroke. J Hand Ther. 2013;26(2):124-30.

4. Santisteban L, Térémetz M, Bleton JP, Baron JC, Maier MA, Lindberg PG. Upper limb outcome measures used in stroke rehabilitation studies: a systematic literature review. PLoS One. 2016;11(5):e0154792.

5. Miller EL, Murray L, Richards L, Zorowitz RD, Bakas T, Clark P, et al. Comprehensive overview of nursing and interdisciplinary rehabilitation care of the stroke patient: a scientific statement from the American Heart Association. Stroke. 2010;41(10):2402-48.

6. Andrianesis $\mathrm{K}$, Tzes A. Development and control of a multifunctional prosthetic hand with shape memory alloy actuators. J Intell Robot Syst. 2015:78:257-89.

7. French B, Leathley MJ, Sutton CJ, Mcadam J, Thomas LH, Forster A, et al. A systematic review of repetitive task training with modelling of resource use, costs and effectiveness. Health Technol Assess. 2008;12(30): iii, ix-x, 1-117.

8. Heiser RD, O'brien V, Schwartz DA. Joint mobilization in the distal upper extremity-putting evidence into practice. J Hand Ther. 2014;27(3):e5.

9. Maitland G, Hengeveld E, Banks K, English K. Maitland's vertebral manipulation. 7th ed. Philadelphia: Butterworth-Heinemann Elsevier; 2005.

10. Pollock A, Farmer SE, Brady MC, Langhorne P, Mead GE, Mehrholz J, et al. Interventions for improving upper limb function after stroke. Cochrane Database Syst Rev. 2014;11:CD010820.

11. Kim YH, Jang HJ, Kim SY. Effect of hip joint mobilization on hip mobility, balance and gait with stroke patients. Phys Ther Korea. 2014;21(2):8-17.

12. Smedes F, Van Der Salm A, Koel G, Oosterveld F. Manual mobilization of the wrist: a pilot study in rehabilitation of patients with a chronic hemiplegic hand post-stroke. J Hand Ther. 2014;27(3):209-15.

13. Park SJ, Youn PS. The immediate effect of wrist joint mobilization with taping on range of motion, grip strength, spasticity in stroke patients. J Kor Phys Ther. 2017;29(4):187-93.

14. Bohannon RW, Smith MB. Interrater reliability of a modified Ashworth scale of muscle spasticity. Phys Ther. 1987;67(2):206-7.

15. Folstein MF, Folstein SE, Mchugh PR. "Mini-mental state": a practical method for grading the cognitive state of patients for the clinician. J Psychiatr Res. 1975;12(3):189-98.

16. Jebsen RH, Taylor N, Trieschmann R, Trotter MJ, Howard LA. An objective and standardized test of hand function. Arch Phys Med Rehabil. 1969;50(6): 311-9.

17. Poole JL. Measures of hand function: arthritis hand function test (AHFT), Australian canadian osteoarthritis hand index (AUSCAN), Cochin hand function Scale, functional index for hand osteoarthritis (FIHOA), Grip Ability Test (GAT), Jebsen hand function test (JHFT), and Michigan hand outcomes questionnaire (MHQ). Arthritis Care Res (Hoboken). 2011;63(11):S189-99.

18. Arshad HS, Shah $\mathbb{H}$, Nasir RH. Comparison of mulligan mobilization with movement and end-range mobilization following Maitland techniques in patients with frozen shoulder in improving range of motion. Pain. 2015;1:2.
19. Khamwong P, Nosaka K, Pirunsan U, Paungmali A. Reliability of muscle function and sensory perception measurements of the wrist extensors. Physiother Theory Pract. 2010;26(6):408-15.

20. Bertrand AM, Fournier K, Brasey MG, Kaiser ML, Frischknecht R, Diserens K. Reliability of maximal grip strength measurements and grip strength recovery following a stroke. J Hand Ther. 2015;28(4):356-62.

21. Carr JH, Shepherd RB. neurological rehabilitation: optimizing motor performance. $2^{\text {nd }}$ ed. New York: Churchill Livingstone Elsevier;2010.

22. Hengeveld E, Banks K. Maitland's Peripheral Manipulation: Management of Neuromusculoskeletal Disorders. $5^{\text {th }}$ ed. London: Churchill Livingstone Elsevier;2014.

23. Kisner C Colby L. Therapeutic exercise: foundations and techniques. $5^{\text {th }}$ ed. Philadelphia: FA. Davis Company; 2007.

24. Hoch MC, Andreatta RD, Mullineaux DR, English RA, Medina McKeon JM, Mattacola CG, et al. Two-week joint mobilization intervention improves selfreported function, range of motion, and dynamic balance in those with chronic ankle instability. J Ortho Res. 2012;30(11):1798-804.

25. Ganesh GS, Mohanty P, Pattnaik M, Mishra C. Effectiveness of mobilization therapy and exercises in mechanical neck pain. Physiother Theory Pract. 2015:31(2):99-106.

26. Lee HS, Park SE, Lee SB, Shin HJ, Kim HR, Choi YD, et al. Effect of ankle joint mobilization and FES on change of ankle movement and the quality of gait in patients with hemiplegia. J Int Acad Phys Ther Res. 2014;5(2):738-42.

27. Kluding P, Zipp GP. Effect of ankle joint mobilization on ankle mobility and sit-to-stand in subjects with hemiplegia. J Neurol Phys Ther. 2004;28(2):7283.

28. An CM, Won Jl. Effects of ankle joint mobilization with movement and weight-bearing exercise on knee strength, ankle range of motion, and gait velocity in patients with stroke: a pilot study. J Phys Ther Sci. 2016;28(2): 689-94.

29. Hunter SM, Crome P, Sim J, Pomeroy VM. Effects of mobilization and tactile stimulation on recovery of the hemiplegic upper limb: a series of replicated single-system studies. Arch Phys Med Rehabil. 2008;89(10):2003-10.

\section{Publisher's Note}

Springer Nature remains neutral with regard to jurisdictional claims in published maps and institutional affiliations.

\section{Submit your manuscript to a SpringerOpen ${ }^{\odot}$ journal and benefit from:}

- Convenient online submission

- Rigorous peer review

- Open access: articles freely available online

- High visibility within the field

- Retaining the copyright to your article

Submit your next manuscript at $\boldsymbol{\nabla}$ springeropen.com 